\title{
Up-regulation of nitric oxide synthases by erythropoietin alone or in conjunction with ischemic preconditioning in ischemia reperfusion injury of rat kidneys
}

\author{
Mohammed Elshiekh ${ }^{1}$, Mehri Kadkhodaee ${ }^{2}$, Behjat Seifi ${ }^{2}$, Mina Ranjbaran ${ }^{2}$ and Hassan Askari ${ }^{2}$ \\ ${ }^{1}$ Department of Physiology, Faculty of Medicine, Tehran University of Medical Sciences, International campus, Tehran, \\ Iran \\ ${ }^{2}$ Department of Physiology, Faculty of Medicine, Tehran University of Medical Sciences, Tehran, Iran
}

\begin{abstract}
The effects of erythropoietin (EPO) alone or in conjunction with ischemic preconditioning (IPC) on nitric oxide synthase as well as comparing their effects on oxidative stress and proinflammatory cytokines are studied. Rats underwent bilateral renal ischemia of $50 \mathrm{~min}$ followed by $24 \mathrm{~h}$ reperfusion. They were administered EPO (5000 iu/kg i.p.) and/or subjected to IPC and sacrificed after $24 \mathrm{~h}$, then plasma and tissue samples were obtained. Treatment of either EPO or IPC and their combination attenuates oxidative stress, decreases histological damages, inhibits proinflammatory response, and up-regulates iNOS and eNOS gene expression compared to IR group. In addition, $\mathrm{EPO}+\mathrm{IPC}$ and $\mathrm{EPO}$ treatment produced significant up-regulation in iNOS gene expression compared to IPC group. In IPC and EPO+IPC groups, more powerful effect on up-regulation of eNOS gene expression was shown compared to EPO group. Our findings suggest that treatment with EPO or IPC and their combination improve renal function and preserve tubular damage induced by IR injury. These advantageous effects were closely related to reducing oxidative stress, suppressing proinflammatory response and enhancing generation of NO. IPC was more powerful in enhancement of eNOS gene expression compared to EPO that was more effective in increasing of iNOS gene expression.
\end{abstract}

Key words: Ischemia reperfusion - Oxidative stress - Proinflammatory response - iNOS - eNOS

\section{Introduction}

Renal ischemia-reperfusion (IR) injury, an inflammatory pathophysiological process, leads to acute kidney failure, delayed graft function, and early mortality in patients subjected to kidney transplantation (Kosieradzki and Rowinski 2008). Several factors have crucial roles in the pathophysiology of IR injury, such as vascular endothelium, leukocytes, reactive oxygen species and adhesion molecules as well as inflammatory mediators (Elahi et al. 2009). Overproduction of ROS results in an increase in lipid peroxidation (MDA) by devastated unsaturated fatty acids in the cell membrane producing a decrease in endogenous antioxidants enzymes such as SOD (Wang et al. 2013).

Correspondence to: Mehri Kadkhodaee, Department of Physiology, Faculty of Medicine, Tehran University of Medical Sciences, Tehran 1417613151, Iran

E-mail: kadkhodm@tums.ac.ir
Nitric oxide (NO) as a biological mediator plays an important role in a variety of biological processes and is a fundamental component in the fields of biochemistry, physiology, immunology and neuroscience (Omer et al. 2012). NO is known as an essential mediator of physiological and pathological processes of renal IR injury (LopezMarti et al. 2003). NO is produced from L-arginine by the enzyme nitric oxide synthase (NOS), which exists in three forms. Two are constitutive, neuronal nitric oxide synthase (nNOS, also known as NOS I) and endothelial nitric oxide synthase (eNOS, also known as NOS III) and one is inducible (iNOS, also known as NOS II, Ignarro 2000). Recent studies suggest the presence of a potentially new isoform of NOS in mitochondria (mtNOS) which was characterized as a constitutive NOS isoform (Giulivi et al. 1998; Ghafourifar et al. 1999). NO regulates neutrophil recruitment by suppressing the expression of adhesion molecules in the vascular endothelium producing increased blood flow to ischemic regions (Laroux et al. 2001). 
Erythropoietin (EPO) is a complex molecule, which regulates red blood cell production in the bone marrow. Recombinant human EPO (rHuEPO) is commercially available and is widely used for the treatment of anemia (Boissel and Cerami 1993). EPO has been shown to protect different organs including brain, heart, and kidney against IR injury (Brines et al. 2008). EPO mediates cytoprotective effects by regulating a variety of signal pathways, which comprise mitogen-activated protein kinase (MAPK) and phosphatidylinositol 3-kinase (PI3K)/Akt (Li et al. 2013; Kwon et al. 2014). It has been documented that PI3K/Akt signaling pathway activation exerts protective effects on IR, as activated Akt increases the expression of eNOS and the generation of NO in endothelial cells (Yao et al. 2013). Nevertheless, the cytoprotective mechanisms underlying the effects of EPO on renal IR injury remain to be fully elucidated.

Ischemic preconditioning (IPC), known as brief sporadic episodes of ischemia alternating with reperfusion, increases the tolerance of the ischemic kidneys against sustained IR injury (Hausenloy and Yellon 2009). Despite intensive investigations, the mechanisms mediating this protective effect are still unclear. However, it has been documented that the benefits of IPC may be mediated by the synthesis of vasoactive mediators, such as nitric oxide (Park et al. 2003). In previous study, we reported the ameliorative effect of EPO and IPC individually on renal IR injury in rats. In that study both treatments have been able to ameliorate renal dysfunction and oxidative stress in kidney tissues (Elshiekh et al. 2015). In the current study, we investigated the effects of EPO alone or in conjunction with IPC on different nitric oxide synthases gene expression as well as comparing their effects on oxidative stress and proinflammatory cytokines.

\section{Materials and Methods}

\section{Animals}

Male Wistar albino weighing 230-300 g were provided by Tehran University of Medical Sciences. Rats were kept in ordinary group cages in a temperature-controlled $\left(21 \pm 1^{\circ} \mathrm{C}\right)$ with a $12 / 12 \mathrm{~h}$ light/dark cycle. They were fed with a standard rat chow and allowed to drink water ad libitum. The study protocol was reviewed and approved by the Animal Care and Use Committee of the Tehran University of Medical Sciences, School of Medicine.

\section{Surgical procedures}

Rats were anesthetized by an intraperitoneal administration of ketamine $(50 \mathrm{mg} / \mathrm{kg})$ and xylazine $(10 \mathrm{mg} / \mathrm{kg})$ and placed on a heating pad in order to maintain their body temperature at $37 \pm 1^{\circ} \mathrm{C}$. A tail-cuff was connected to a pulse transducer device (MLT125/R; AD Instruments, Castle Hill, NSW, Australia) for the measurement of systolic blood pressure and heart rate. The transducer was linked to a PowerLab/4SP data-acquisition system (Chart, version 5; AD Instruments). Based on this technique, rats with blood pressure below $60 \mathrm{mmHg}$ were excluded from the study. A midline laparotomy was performed, in which the abdominal cavity was fully exposed. Bilateral renal pedicles were isolated carefully and blood supply to both kidneys was interrupted by clamping renal vascular pedicles with a noncrushing microvascular clamp. Reperfusion was performed after $50 \mathrm{~min}$ ischemia to allow return of blood flow to the kidneys for 24 hours. The edges of the abdominal incision were sutured in two layers.

IPC was performed by three cycles of alternating 3 minutes of bilateral renal pedicles ischemia and 3 minutes reperfusion. The occlusion was achieved by non-crushing microvascular clamp.

\section{Study design}

Animals were allocated randomly into five groups:

IR group $(n=6)$ : rats were underwent $50 \mathrm{~min}$ ischemia followed by $24 \mathrm{~h}$ reperfusion,

Sham group $(n=6)$ : rats were underwent same surgical procedures without induction of bilateral IR,

EPO group $(n=6)$ : rats were administered EPO $5000 \mathrm{iu} /$ $\mathrm{kg}$ i.p. $30 \mathrm{~min}$ before IR,

IPC group $(n=6)$ : similar to IR group but, 3 cycles of 3 min ischemia followed by 3 min reperfusion (IPC method) were performed before induction of IR,

EPO+IPC group $(n=6)$ : similar to IR group but EPO $5000 \mathrm{iu} / \mathrm{kg}$ was administered (30 min before IR) plus IPC was performed before induction of IR.

At the end of the surgery, rats were kept in individual metabolic cages for urine collection over a period of $24 \mathrm{~h}$. After $24 \mathrm{~h}$ reperfusion, they were anesthetized by a mixture of ketamine $(50 \mathrm{mg} / \mathrm{kg})$ and xylazine $(10 \mathrm{mg} / \mathrm{kg})$ and blood samples were collected from the inferior vena cava. Rats were sacrificed and their kidneys were harvested before being washed and dissected in cold normal saline. The kidneys were immediately harvested, either fixed in $10 \%$ formalin for histology or snaps frozen in liquid nitrogen and stored at $-70^{\circ} \mathrm{C}$ for oxidative stress and RT-PCR analysis. EPO was partly a gift from Pooyesh Darou Company.

\section{Measurement of biochemical parameters}

Urine samples were gathered throughout reperfusion period, and the volume produced was recorded. Urine concentrations of creatinine and $\mathrm{Na}^{+}$were measured at the end of reperfusion period and were utilized in conjunction with plasma concentrations to estimate creatinine clearance 
(CCr) and fractional excretion of $\mathrm{Na}^{+}$(FENa) using standard formulae. These were used as indicators of glomerular and tubular function respectively.

\section{Renal oxidative stress assessments}

Renal oxidative stress was evaluated by measuring renal malondialdehyde (MDA, an index for lipid peroxidation) and superoxide dismutase (SOD, an endogenous antioxidant enzyme).

\section{Estimation of inflammatory biomarkers}

Tumor necrosis factor $\alpha$ (TNF- $\alpha$ ) and interleukin 6 (IL-6) were determined in the plasma using a rat TNF- $\alpha$ and IL- 6 ELISA kit (R\&D Systems, Inc, USA) and expressed as pg/ml wet tissue.

\section{Histopathological examination}

A part of each kidney was fixed in $10 \%$ formalin, then routinely treated and embedded in paraffin. Thin slices $(5 \mu \mathrm{m})$ were cut and stained with hematoxylin and eosin (H\&E). Slides were then assessed and images were captured utilizing a digital camera comprised in a light microscope.

\section{Real Time PCR}

Total RNA from kidney tissue samples was prepared with RLT reagent according to the manufacturer's instructions (RNeasy Mini Kit; Qiagen). RNA quality and concentration were determined using the Nanodrop 1000 (Thermo- Scientific, USA). Four micrograms of total RNA was reversetranscribed into cDNA with the use of the PrimeScript RT Master Mix (Takara, Japan) as instructed. Gene amplification was achieved in $0.2 \mu$ l volume microtube in ABI7500 realtime PCR. The reaction mixture comprised $4 \mu \mathrm{l}$ of diluted cDNA, 5 pM of each primer, $10 \mu \mathrm{l}$ of $2 \mathrm{X}$ SYBR green master mixes in a total volume of $20 \mu$ l.

The PCR protocol was accomplished as follows; initial step of $95^{\circ} \mathrm{C}$ for $15 \mathrm{~min}$, amplification step of 40 cycles at $95^{\circ} \mathrm{C}$ for $15 \mathrm{~s}$ followed by $1 \mathrm{~min}$ at $58^{\circ} \mathrm{C}$. This program was followed by analysis of melting curve that was performed with linear heating from $60-90^{\circ} \mathrm{C}$.

Under similar conditions, the PCR assay was performed with HPRT-1 as a house keeping gene using specific primers (Table 1) and considered as internal control.

\section{Statistical analysis}

The software of SPSS version 13 (SPSS Inc, IL, USA) was used for statistical analysis. All values depicted in the text and figures were showed as the mean \pm SEM. Data were analyzed using one-way ANOVA followed by post hoc Tukey's test; $p<0.05$ was considered significant.

\section{Results}

\section{Renal function and histology}

Compared to the sham group, rats that subjected to IR showed a significant decrease in $\mathrm{CCr}$ and a significant increase in FENa $(p<0.05)$. Pretreatment with EPO and IPC produced a significant protective effect against the IR-induced deterioration of renal function $(p<0.05)$. However, EPO treatment along with IPC showed a better improvement, although not statistically significant, in the markers of renal function compared to the other treated groups (Figure 1).

Sham group had normal histology by light microscopy. In the IR group, the sections showed severe damage including frequent cast formation in the tubules. Cellular disintegration was seen especially in the mid-cortical and corticomedullary tubules. These features were compatible with necrotic changes. In the tissues of IPC group, less tubular damage was seen. Although, some tubular debris were also present. The same histological characteristics applied to EPO group in which moderate improvement was seen comparing to IR group. However, these sections were not different from IPC tissues. In the EPO+IPC group, there was more improvement comparing to the other two treatment groups.

Table 1. Primers used for Real-Time PCR analysis

\begin{tabular}{|c|c|c|}
\hline Gene & & Specific primers \\
\hline \multirow{2}{*}{ iNOS } & Sense Strand Sequence & TCGCTGGTTTGAAACTTCTCAG \\
\hline & Anti-Sense Strand Sequence & CTCCGTGGGGCTTGTAGTTGA \\
\hline \multirow{2}{*}{ eNOS } & Sense Strand Sequence & GGCTGAGTACCCAAGCTGAG \\
\hline & Anti-Sense Strand Sequence & ATTGTGGCTCGGGTGGATTT \\
\hline \multirow{2}{*}{ HPRT-1 } & Sense Strand Sequence & CTCCTCAGACCGCTTTTCCC \\
\hline & Anti-Sense Strand Sequence & AGCAAGTCTTTCAGTCCTGTCC \\
\hline
\end{tabular}


A

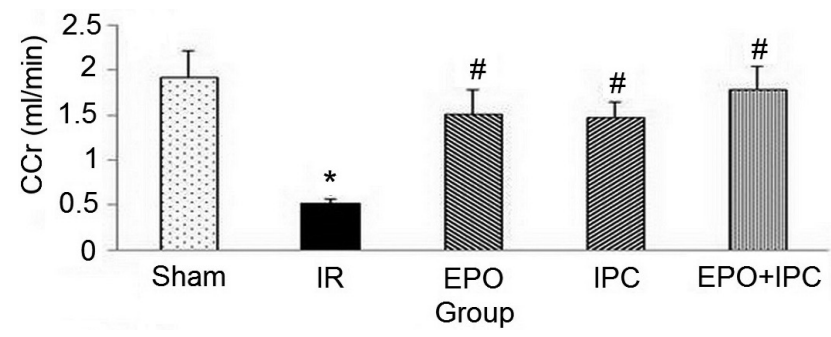

B

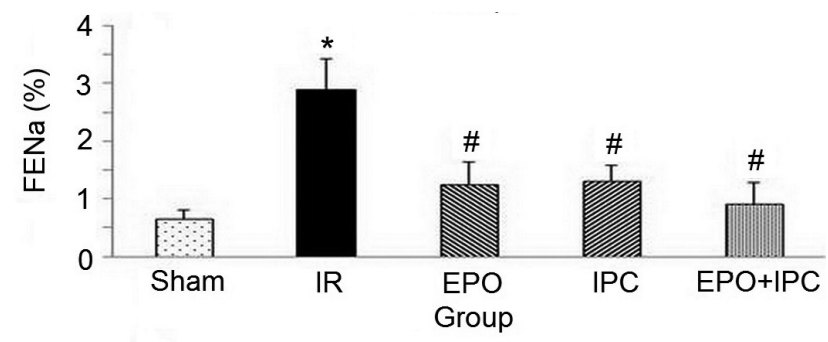

Figure 1. Effect of erythropoietin (EPO) and/or ischemic preconditioning (IPC) on renal function markers: creatinine clearance $(\mathrm{CCr} ; \mathbf{A})$ and fractional excretion of sodium (FENa; B). Data represent mean \pm SEM $(n=6){ }^{*} p<0.05 v s$. Sham group, \# $p<$ $0.05 v s$. IR group. IR group, rats were subjected to renal ischemia followed by reperfusion; EPO group, rats treated with EPO before IR procedure; IPC group, rats were subjected to ischemic preconditioning before IR procedure; EPO+IPC group, rats treated with $\mathrm{EPO}$ and IPC before IR procedure.

No obvious necrosis was detected and less luminal debris was observed. (Figure 2).

\section{Renal oxidative stress}

Rats underwent renal IR showed a significant raise in renal MDA content, and a significant decrease in renal SOD activity $(p<0.05)$. Pretreatment with EPO and/or IPC revealed a significant reduction in renal MDA content and a significant increase in renal SOD activity compared to IR group $(p<0.05)$. (Figure $3 \mathrm{~A}$ and $\mathrm{B})$.

\section{TNF- $\alpha$ and IL-6}

Rats underwent renal IR showed a substantial increase in plasma levels of TNF- $\alpha$ and IL-6, compared to sham group $(p<0.05)$. All three treatment groups showed significant decreases in plasma levels of TNF- $\alpha$ and IL-6, compared to IR group $(p<0.05)$. However, in the combination group the reduction in plasma TNF- $\alpha$ was more prominent so its level was not significantly different from the sham group. (Figure 3C and D).
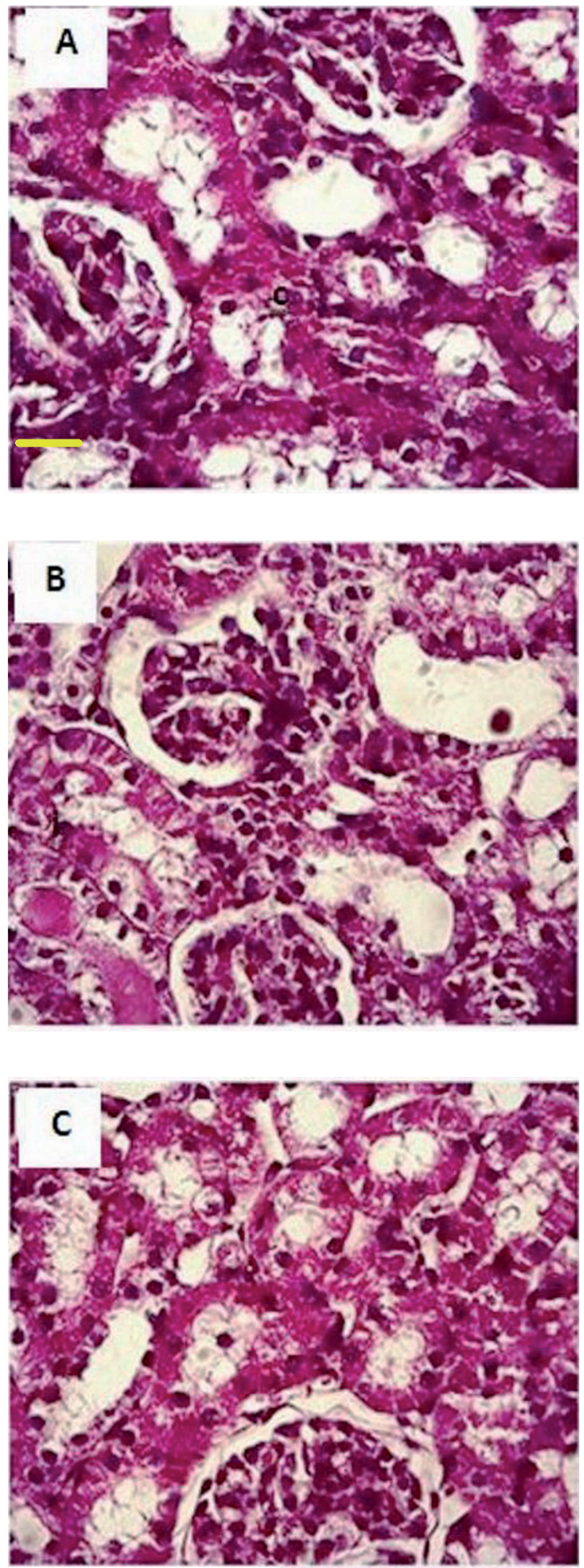

Figure. 2. Effect of erythropoietin (EPO) and/or ischemic preconditioning (IPC) on renal histological changes induced by renal ischemia reperfusion in EPO group (A), IPC group (B) and EPO+IPC group $(\mathbf{C})$. Kidney sections were stained with hematoxylin and eosin (H\&E, scale bar: $100 \mu \mathrm{m}$, magnification: $\times 400$ ). 


\section{iNOS and eNOS expression}

Real time PCR showed a significant increase in iNOS gene expression in all studied groups (IR, EPO, IPC and EPO+IPC) compared to sham group $(p<0.05)$. There is no difference in eNOS gene expression between IR and sham groups. Furthermore, EPO pretreatment or IPC and their combination showed a significant increase in iNOS and eNOS gene expression compared to IR group $(p<0.05)$. $\mathrm{EPO}+\mathrm{IPC}$ and EPO groups showed significant increases in iNOS gene expression compared to IPC group $(p<0.05)$. In addition, EPO+IPC and IPC groups showed a significant increase in the eNOS gene expression compared to EPO group $(p<0.05)$ (Figure $4 A$ and B).

\section{Discussion}

In the present study, we have investigated the effects of EPO alone or in conjunction with IPC on NOS gene expression as well as comparing their effect on oxidative stress and proinflammatory cytokines. We demonstrated that pretreat- ment with either EPO or IPC was equipotent in ameliorating renal damage and dysfunction. In addition, we demonstrated that both of the treatments and their combination could effectively attenuate renal injury in rat kidney IR model. The protective effects of EPO and IPC were associated with enhanced up-regulation of iNOS and eNOS gene expression.

NO is suggested to have crucial role in normal and pathophysiological process of renal IR (Lopez et al. 2003). It down-regulates inflammatory reactions, which are the main contributors to IR injury (Laroux et al. 2001). Furthermore, NO regulates neutrophil recruitment by suppressing the expression of adhesion molecules in the vascular endothelium causing increased blood flow to the ischemic regions. NO generation is mediated by the activities of NOS enzymes, whose expression is, in turn, altered by signaling pathways involved in inflammation, such as NF-B and MAPK (Hickey et al. 2001; Laroux et al. 2001). Vinas and co-workers documented that NO may have a protective effect due to its anti-apoptotic action to decrease leukocyte-endothelial interactions. NO formation can result in vasodilatation and inhibition of platelet plug formation, as well as reduction of the inflammatory response (Vinas et al. 2006). Thus, protec-
A

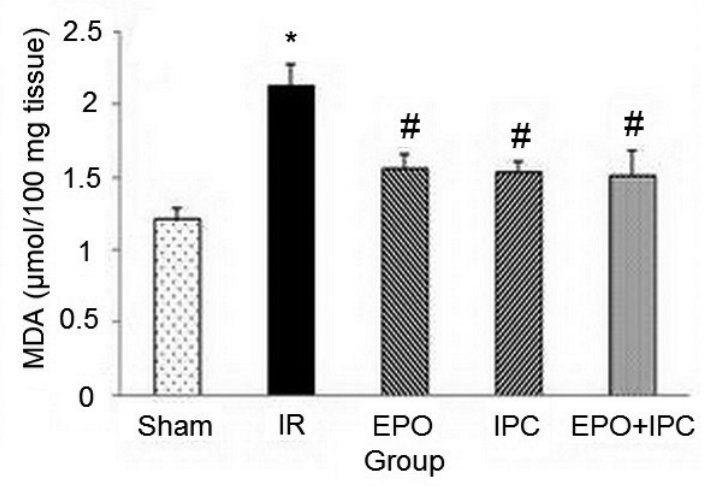

C

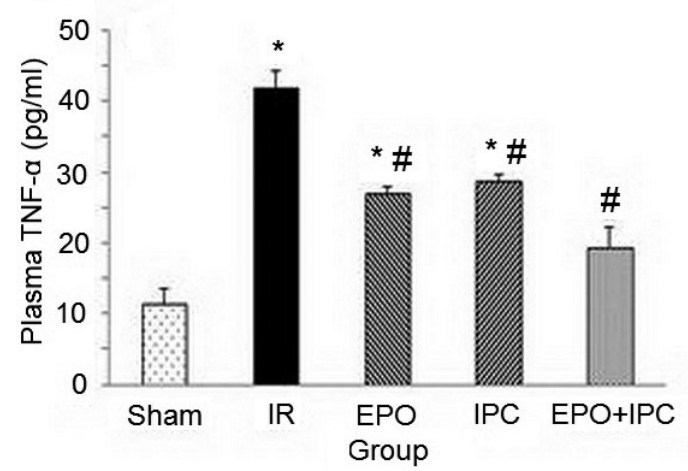

B

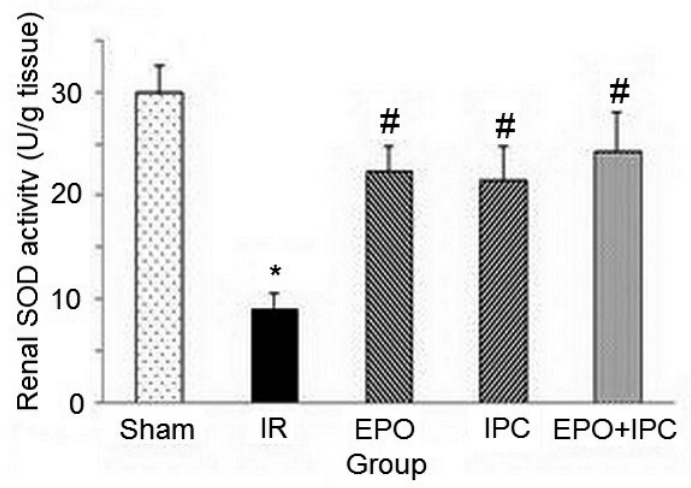

D

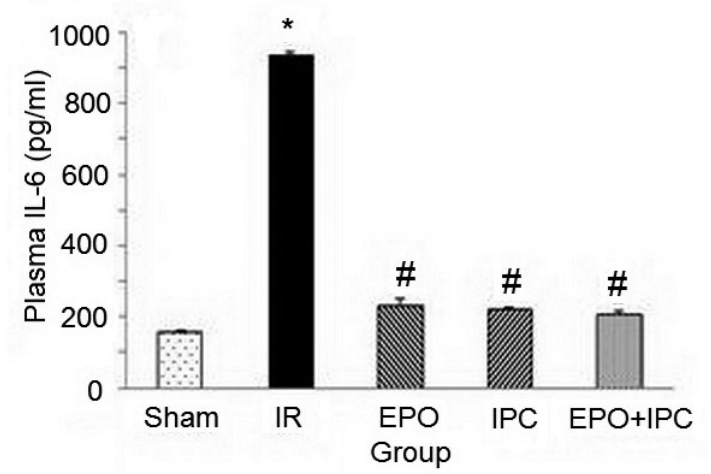

Figure 3. Effect of EPO and/or IPC on renal malondialdehyde (MDA) content (A), renal superoxide dismutase (SOD) activity (B), tumor necrosis factor (TNF- $\alpha)($ C) and plasma interleukin 6 (IL-6) (D). Data represent mean \pm SEM $(n=6)$; $p<0.05 v s$. Sham group, \# $p<$ $0.05 v s$. IR group. (For abbreviations, see Figure 1). 
A

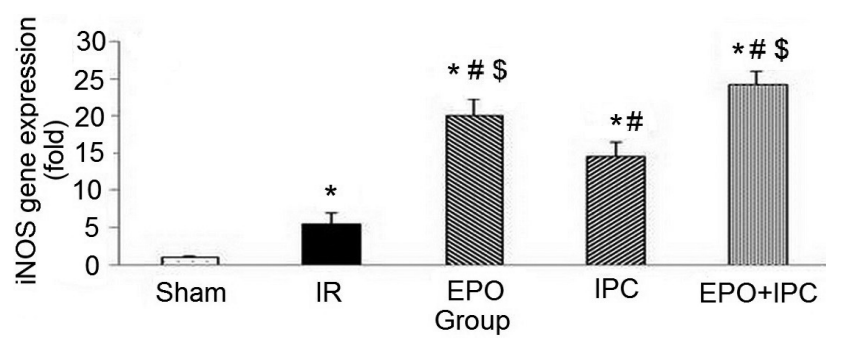

B

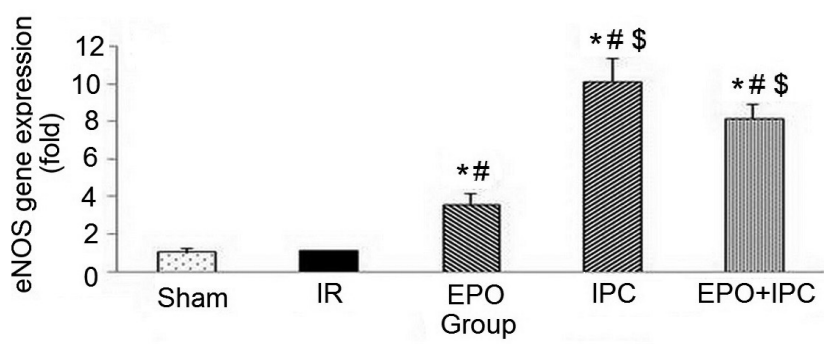

Figure 4. Effect of EPO and/or IPC on iNOS (A) and eNOS (B) gene expression. Data represent mean $\pm \operatorname{SEM}(n=6) ;{ }^{*} p<0.05 v s$. Sham group, $\# p<0.05 v s$. IR group, $\$ p<0.05 v s$. treatment groups (EPO, IPC, EPO+IPC). (For abbreviations, see Figure 1).

tive effects of NO may depend on its concentration, site of release and duration of action (Goligorsky et al. 2004).

In this study, there was a significant increase in iNOS gene expression in IR group compared to sham animals and this enhancement was even higher in the treatment groups. Study by Torras et al. (2002) documented that NOS-generated NO supressed IR-induced renal damage and the beneficial effect of preconditioning is related to the local production of NO. It seems that the defense system of the body has started to protect the kidney from IR injury by enhancement of NO production through inducing NOS enzyme activity and this protective effect has been reinforced by treatments.

The effect of IPC alone or in combination with EPO was more powerful in enhancement of eNOS gene expression than EPO alone. Furthermore, the present study demonstrated more enhancement in the gene expression of iNOS by EPO treatment and combination than IPC alone. There is evidence that $\mathrm{EPO}$ stimulates the phosphorylation of serine residues on eNOS, resulting in its activation (Mastromarino et al. 2011). EPO mediates cytoprotective effects by regulating a variety of signal pathways, which comprise MAPKs and PI3K/Akt (Li et al. 2013; Kwon et al. 2014). It has been documented that PI3K/Akt signaling pathway activation exerts protective effects on IR, as activated Akt increases the expression of eNOS and the generation of NO in endothelial cells (Yao et al. 2013). Recently, Ge et al. (2015) demonstrated that in rats, IPC contributes to the early restoration of renal affective blood flow (RABF), probably through eNOS and iNOS-mediated NO production thereby alleviating IRinduced renal dysfunction and histological damage. IPC, known as brief sporadic episodes of ischemia alternating with reperfusion, increases the tolerance of the ischemic kidneys against sustained IR injury (Hausenloy et al. 2009). Despite intensive investigations, the mechanisms responsible for this protective effect is still unclear. However, it has been documented that the benefits of IPC may be mediated by the synthesis of vasoactive mediators, such as NO (Park et al. 2003). In this study, we demonstrated that IPC, either alone or in combination with EPO, increased $\mathrm{NNOS}$ and $\mathrm{eNOS}$ gene expression comparing with IR group. IPC was more powerful in enhancement of eNOS gene expression compared to EPO. Barrier et al. (2005) reported that IPC stimulates the production of NO through up-regulation of iNOS gene expression. In addition, activation of eNOS is beneficial as the enhanced production of $\mathrm{NO}$ results in local vasodilation, suppresses adhesion of platelets and neutrophils, and regulates angiogenesis (Tyml 2011). Factors that release NO or reinforce the generation of endogenous NO may attenuate excessive intra-renal vasoconstriction and reduce renal dysfunction (Kwon et al. 2009). Thus, up-regulation of iNOS and eNOS mediated by EPO and IPC may contribute to the beneficial effects of EPO and IPC which was reported here.

Renal ischemia reperfusion injury resulted in an increased generation of proinflammatory cytokines such as TNF- $\alpha$ and IL-6 (Rezende-Neto et al. 2002). TNF- $\alpha$ mediates activation of endothelium to express adhesion molecules and additional proinflammatory molecules. These mediators arouse the infiltration of cells into tubulointerstitium to encourage further inflammation and tissue deterioration (Ysebaert et al. 2000). In the present study, plasma TNF- $\alpha$ and IL- 6 were noted to be markedly increased in the animals subjected to IR injury compared to sham rats. These results further substantiate that IR-associated proinflammatory response may contribute to the pathogenesis of renal IR injury. Treatment with EPO and/ or IPC significantly attenuated IR-induced increase in plasma TNF- $\alpha$ and IL- 6 level. Thus it may be suggested that the renoprotective effects of EPO and IPC against renal damage and dysfunction might be associated with their actions in reducing circulating proinflammatory cytokines. These findings are in agreement with Ates et al. (2005) which documented that EPO treatment suppresses renal inflammation during IR damage by reducing proinflammatory cytokines, TNF- $\alpha$ and IL- 6 activation. Therefore, inhibition of inflammatory response may be the potential mechanisms of reno-protective effects of EPO and IPC against renal IR injury.

In the current study, we demonstrated that treatment with EPO or IPC and their combination produced a substantial improve in tubular and glomerular function as pointed by 
a marked increase in CCr, and substantial decrease in FENa and changes in histologic features compared to the IR group. Our findings of a protective effect of EPO pretreatment in IR injury are in good agreement with previous study (Zhang et al. 2015) demonstrating the beneficial effect of EPO pretreatment in improving renal function and histology. Tsutsui et al. (2013) reported that IPC induced substantial improvement in renal function markers and histological features. In the present study, the results from EPO+IPC group, namely cast formation, cellular disintegration in mid-cortical and corticomedullary tubules, tubular damage and necrosis were significantly less compared to rats treated with EPO or IPC alone.

Regarding oxidative stress, our present study revealed that $50 \mathrm{~min}$ ischemia followed by $24 \mathrm{~h}$ reperfusion produced a marked decrease in SOD activity. These findings are in agreement with previous reports demonstrating that IR induces oxidative stress as evidenced by decreasing SOD activity and increasing MDA content (Sheridan and Bonventre 2001). In the present study we demonstrated that treatment with EPO or IPC and their combination markedly ameliorated oxidative stress by increasing SOD activity and consequently by decreasing MDA content. In consistent with our study, administration of EPO along with sodium selenite decreased oxidative stress and activated PI3K/NO signaling pathway (Liu et al. 2015) in a rat ischemia-reperfusion induced renal injury model. Shokeir et al. (2015) documented that combination of IPC and sulforaphone offered more improvements in the antioxidant enzymes and inflammatory state, but not the apoptotic markers.

In conclusion, our findings suggested that treatment with EPO and/or IPC improve renal function and decrease tubular damage induced by IR. These advantageous effects were closely related to reducing oxidative stress, suppressing proinflammatory response and enhancing generation of NO via up-regulation of iNOS and eNOS gene expression. In addition, our findings demonstrate that up-regulation of iNOS and eNOS are essential for EPO- and IPC-mediated amelioration of renal function in IR injury.

Acknowledgement. This work was supported by a grant from the International Campus of Tehran University of Medical Sciences (No 27596).

Conflict of interest. On behalf of all authors, the corresponding author states that there is no conflict of interest.

\section{References}

Ates E., Yalcin A. U., Yilmaz S., Koken T., Tokyol C. (2005): Protective effect of erythropoietin on renal ischemia and reperfusion injury. ANZ J. Surg. 75, 1100-1105

https://doi.org/10.1111/j.1445-2197.2005.03612.x
Barrier A., Olaya N., Chiappini F., Roser F., Scatton O., Artus C., Franc B., Dudoit S., Flahault A., Debuire B., Azoulay D., Lemoine A. (2005): Ischemic preconditioning modulates the expression of several genes, leading to the overproduction of IL-1Ra, iNOS, and Bcl-2 in a human model of liver ischemiareperfusion. FASEB J. 19, 1617-1626 https://doi.org/10.1096/fj.04-3445com

Boissel J. P., Lee W. R., Presnell S. R., Cohen F. E., Bunn H. F. (1993): Erythropoietin structure-function relationships. Mutant proteins that test a model of tertiary structure. J. Biol. Chem. 268, 15983-15993

Brines M., Cerami A. (2008): Erythropoietin-mediated tissue protection: reducing collateral damage from the primary injury response. J. Int. Med. 264, 405-432 https://doi.org/10.1111/j.1365-2796.2008.02024.x

Elahi M. M., Kong Y. X., Matata B. M. (2009): Oxidative stress as a mediator of cardiovascular disease. Oxid. Med. Cell Longev. $2,259-269$ https://doi.org/10.4161/oxim.2.5.9441

Elshiekh M., Kadkhodaee M., Seifi B., Ranjbaran M., Ahghari P. (2015): Ameliorative effect of recombinant human erythropoietin and ischemic preconditioning on renal ischemia reperfusion injury in rats. Nephro. Urol. Mon. 7, e31152 https://doi.org/10.5812/numonthly.31152

Ge Y. Z., Wu R., Xin H., Liu H., Lu T. Z., Zhao Y. C., Shen J. W., Hu Z. K., Yu P., Zhou L. H., Xu L. W, et al. (2015): Effects of ischemic preconditioning on the systemic and renal hemodynamic changes in renal ischemia reperfusion injury. Int. J. Clin. Exp. Pathol. 1, 1128-1140

Ghafourifar P., Schenk U., Klein S. D., Richter C. (1999): Mitochondrial nitric oxide stimulation causes cytochrome $\mathrm{C}$ release from isolated mitochondria. Evidence for intramitochondrial peroxynitrite formation. J. Biol. Chem. 274, 31185-31188 https://doi.org/10.1074/jbc.274.44.31185

Giulivi C., Poderoso J. J., Boveris A. (1998): Production of NO by mitochondria. J. Biol. Chem. 273, 11038-11043 https://doi.org/10.1074/jbc.273.18.11038

Goligorsky M. S., Brodsky J. V., Noiri E. (2004): NO bioavaibility, endothelial dysfunction, and acute renal failure: new insights in pathophysiology. Semin. Nephrol. 24, 316-323

https://doi.org/10.1016/j.semnephrol.2004.04.003

Hausenloy D. J., Yellon D. M. (2009): Preconditioning and postconditioning: underlying mechanisms and clinical application. Atherosclerosis 204, 334-341 https://doi.org/10.1016/j.atherosclerosis.2008.10.029

Hickey M. J., Granger D. N., Kubes P. (2001): Inducible nitric oxide synthase (iNOS) and regulation of leucocyte/endothelial cell interactions: studies in iNOS-deficient mice. Acta Physiol. (Scand) 173, 119-126

https://doi.org/10.1046/j.1365-201X.2001.00892.x

Ignarro L. J. (2000): Nitric Oxide: Biology and Pathophysiology. Academic press, San Diego

Kosieradzki M., Rowinski W. (2008): Ischemia/reperfusion injury in kidney transplantation: mechanisms and prevention. Transplant. Proc. 40, 3279-3288

https://doi.org/10.1016/j.transproceed.2008.10.004

Kwon M. S., Kim M. H., Kim S. H., Park K. D., Yoo S. H., Oh I. U., Pak S., Seo Y. J. (2014): Erythropoietin exerts cell protective 
effect by activating PI3K/Akt and MAPK pathways in C6 Cells. Neurol. Res. 36, 215-223 https://doi.org/10.1179/1743132813Y.0000000284

Kwon O., Hong S. M., Ramesh G. (2009): Diminished NO generation by injured endothelium and loss of macula densa nNOS may contribute to sustained acute kidney injury after ischemiareperfusion. Am. J. Physiol. Renal. Physiol. 296, F25-33 https://doi.org/10.1152/ajprenal.90531.2008

Laroux F. S., Pavlick K. P., Hines I. N., Kawachi S., Harada H., Bharwani S., Hoffman J. M., Grisham M. B. (2001): Role of nitric oxide in inflammation. Acta Physiol. (Scand) 173, 113-118 https://doi.org/10.1046/j.1365-201X.2001.00891.x

Liu L., Liu C., Hou L., Lv J., Wu F., Yang X., Ren S., Ji W., Wang M., Chen L. (2015): Protection against ischemia/reperfusion-induced renal injury by cotreatment with erythropoietin and sodium selenite. Mol. Med. Rep. 12, 7933-7940

Li X. J., Zhang G. X., Sun N., Sun Y., Yang L. Z., Du Y. J. (2013): Protective effects of erythropoietin on endotoxin-related organ injury in rats. J. Huazhong Univ. Sci. Technolog. Med. Sci. 33, 680-686 https://doi.org/10.1007/s11596-013-1180-1

Lopez-Marti J., Sola A., Pi F., Alfaro V., Marco A., Hotter G. (2003): Nucleotides modulate renal ischemiaereperfusion injury by different effects on nitric oxide and superoxide. Clin. Exp. Pharmacol. Physiol. 30, 242-248

https://doi.org/10.1046/j.1440-1681.2003.03821.x

Mastromarino V., Volpe M., Musumeci M. B., Autore C., Conti E. (2011): Erythropoietin and the heart: Facts and perspectives. Clin. Sci. (Lond) 120, 51-63 https://doi.org/10.1042/CS20100305

Omer N., Rohilla A., Rohilla S., Kushnoor A. (2012): Nitric oxide: role in human biology. Int. J. Pharm. Sci. Drug Res. 4, 105-109

Park K. M., Byun J. Y., Kramers C. (2003): Inducible nitric-oxide synthase is an important contributor to prolonged protective effects of ischemic preconditioning in the mouse kidney. J. Biol. Chem. 278, 27256-27266 https://doi.org/10.1074/jbc.M301778200

Rezende-Neto J. B., Moore E. E., Melo de Andrade M. V., Teixeira M. M., Lisboa F. A., Arantes R. M., de Souza D. G., da CunhaMelo J. R. (2002): Systemic inflammatory response secondary to abdominal compartment syndrome: Stage for multiple organ failure. J. Trauma 53, 1121-1128 https://doi.org/10.1097/00005373-200212000-00015

Sheridan A. M., Bonventre J. V. (2001): Pathophysiology of ischemic acute renal failure. Contrib. Nephrol. 132, 7-21 https://doi.org/10.1159/000060075
Shokeir A. A., Barakat N., Hussein A. M., Awadalla A., Harraz A. M, Khater S., Hemmaid K., Kamal A. I. (2015): Activation of $\mathrm{Nrf2}$ by ischemic preconditioning and sulforaphane in renal ischemia/reperfusion injury: a comparative experimental study. Physiol. Res. 64, 313-323

Torras J., Herrero-Fresneda I., Lloberas N., Riera M., Ma Cruzado J., Ma Grinyó J. (2002): Promising effects of ischemic preconditioning in renal transplantation. Kidney Int. 61, 2218-2227 https://doi.org/10.1046/j.1523-1755.2002.00360.x

Tsutsui H., Tanaka R., Yamagata M., Yukimura T., Ohkita M., Matsumura Y. (2013): Protective effect of ischemic preconditioning on ischemia/reperfusion-induced acute kidney injury through sympathetic nervous system in rats. Eur. J. Pharmacol. 718, 206-212 https://doi.org/10.1016/j.ejphar.2013.08.032

Tyml K. (2011): Critical role for oxidative stress, platelets, and coagulation in capillary blood flow impairment in sepsis. Microcirculation 18, 152-162 https://doi.org/10.1111/j.1549-8719.2010.00080.x

Vinas J. L., Sola A., Genseca M., Alfaro V., Pí F., Hotter G. (2006): $\mathrm{NO}$ and NOS isoforms in the development of apoptosis in renal ischemia/reperfusion. Free Radic. Biol. Med. 40, 992-1003 https://doi.org/10.1016/j.freeradbiomed.2005.10.046

Wang H. B., Li Y. X., Hao Y. J., Wang T. F., Lei Z., Wu Y., Zhao Q. P.,Anq H.,Ma L., Liu J. et al. (2013): Neuroprotective effects of LBP on brain ischemic reperfusion neurodegeneration. Eur. Rev. Med. Pharmacol. Sci. 17, 2760-2765

Yao L., Lu P., Li Y., Yang L., Feng H., Huang Y., Zhang D., Chen J., Zhu D. (2013): Osthole relaxes pulmonary arteries through endothelial phosphatidylinositol 3-kinase/Akt-eNOS-NO signaling pathway in rats. Eur. J. Pharmacol. 699, 23-32 https://doi.org/10.1016/j.ejphar.2012.11.056

Ysebaert D. K., De Greef K. E., Vercauteren S. R., Ghielli M., Verpooten G. A., Eyskens E. J., De Broe M. E. (2000): Identification and kinetics of leukocytes after severe ischemia/reperfusion renal injury. Nephrol. Dial. Transplant. 15, 1562-1574 https://doi.org/10.1093/ndt/15.10.1562

Zhang J., Zou Y., Zhong X., Deng H. D., Pu L., Peng K., Wang L. (2015): Erythropoietin pretreatment ameliorates renal ischaemia-reperfusion injury by activating PI3K/Akt signalling. Nephrology 20, 266-272 https://doi.org/10.1111/nep.12384

Received: June 6, 2016

Final version accepted: July 28, 2016

First published online: May 4, 2017 